\title{
Electrodynamic Tethers for Energy Harvesting and Propulsion on Space Platforms
}

\author{
Sven G. Bilén * and Jesse K. McTernan ${ }^{\dagger}$ \\ The Pennsylvania State University, University Park, PA 16802 \\ Brian E. Gilchrist ${ }^{*}$ and Iverson C. Bell ${ }^{\S}$ \\ The University of Michigan, Ann Arbor, MI 48109 \\ and \\ Nestor R. Voronka** and Robert P. Hoyt ${ }^{\dagger \dagger}$ \\ Tethers Unlimited, Inc., Bothell, WA 98011
}

\begin{abstract}
Electrodynamic tethers can be used to generate power onboard spacecraft orbiting the Earth (or any other planetary body with a magnetic field), essentially "harvesting" energy from the orbital energy of the spacecraft. A key benefit of an electrodynamic tether is the high peak power generation capability for a small impact in spacecraft mass and volume. Reciprocally, the same system can be used to provide orbit-raising propulsion, or change of any other orbital parameter if power is applied to the tether. In this work, the research goal is to develop a better understanding of the power generation capabilities of EDT systems on various scales, and to develop system concepts, key elements, and technology roadmaps for their use. This paper reports on preliminary results for large-to-small, nano-, and picosatellite scales. The results show that EDTs are viable devices for providing power to spacecraft and worthy of further investigation.
\end{abstract}

\section{Introduction}

$\mathrm{H}$ arvesting of power and energy from the space environment offers the promise of reduction in fuel usage as well as extended mission lifetimes and/or enhanced system capability. Apart from solar cells, limited adoption of other energy harvesting devices on satellites has occurred, such as the conversion and recovery of thermal energy. Other untapped resources available to satellites include gravity, the planetary magnetic field, and the surrounding ionosphere. Electrodynamic tethers (EDTs) represent an innovative scheme to harvest energy in the orbital environment from these resources.

EDTs can be used to generate power onboard spacecraft orbiting the Earth (or other planetary bodies with a magnetic field), essentially "harvesting" energy from the orbital energy of the spacecraft. Reciprocally, the same system can be used to provide orbit-raising propulsion or to change inclination if current is supplied to the tether. Power levels on the order of $3.8 \mathrm{~kW}$ and a corresponding drag thrust of $\sim 0.55 \mathrm{~N}$ were demonstrated on the TSS $-1 \mathrm{R}$ Shuttle mission, which deployed a tether $\sim 20 \mathrm{~km}$ long (Figure 1). ${ }^{1}$ Hence, one of the significant promises of EDTs is their ability to generate significant power when other sources are not available (i.e., on the dark side of the Earth) and then thrust when other power is available, essentially recharging an "orbital battery". In addition, power generation and thrusting consume no expendables, which allows increased mission lifetime.

\footnotetext{
* Assoc. Professor of Engineering Design, Electrical Engineering, and Aerospace Engineering, 213 Hammond Bldg., Associate Fellow.

${ }^{\dagger}$ Graduate Student Research Assistant, Aerospace Engineering, 229 Hammond Building, Student Member.

* Professor of Electrical Engineering and Space Science, 1301 Beal Ave., Associate Fellow.

$\S$ Graduate Student Research Assistant, Electrical Engineering, 1301 Beal Ave., Student Member.

** VP \& Chief Technologist, 11711 North Creek Parkway South, Suite D-113, Senior Member.

${ }^{\dagger}$ CEO \& Chief Scientist, 11711 North Creek Parkway South, Suite D-113, Senior Member.
} 
While tether systems (EDT and nonconducting) have flown and many novel configurations have been proposed for accomplishing an array of missions, the use of EDTs as energy-harvesting devices has not been systematically explored and the requisite technology advanced. The goal of our research effort is to develop a better understanding of the power generation capabilities of EDT systems on various scales, and to develop system concepts, key elements, and technology roadmaps for their use. This paper overviews our research effort and the research directions we are pursuing, which include

- Characterizing the scaling of peak and average power available from an EDT system as a function of key variables such as tether length, EDT system mass, orbit altitude and inclination, and

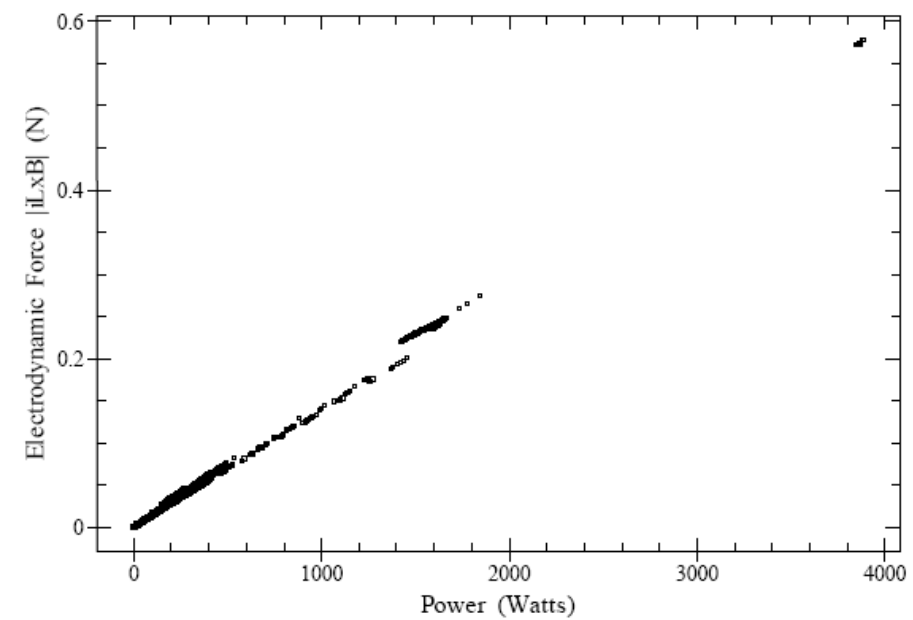

Figure 1. TSS-1R (1996) demonstrated $\sim 3.8 \mathrm{~kW}$ power generation and a maximum electrodynamic drag of $\sim 0.55 \mathrm{~N}$ at tether break. solar conditions;

- Addressing EDT performance parameters such as generated electromotive force (emf), tether current, and altitude change as a function of power generated or applied;

- Developing system concepts for the use of EDTs for power generation on three classes of spacecraft: nanosats $(1-50 \mathrm{~kg})$, microsats $(50-500 \mathrm{~kg})$, and large satellites $(>500 \mathrm{~kg})$ in a variety of orbits; and

- Comparing EDT power generation capabilities and characteristics to alternative technologies such as batteries, ultracapacitors, and flywheels, and quantify mission characteristics where EDTs provide a significant advantage.

We also report on our preliminary results for energy harvesting with EDTs on large-to-small (high power), nano-, and pico-satellite scales

\section{Background}

\section{A. Electrodynamic Tether Principles}

The principles behind the operation of an orbiting EDT system are fairly straightforward. In general, EDTs possess three key properties $\left.{ }^{2}: 1\right)$ the wire has an intrinsic electromotive force $(e m f)$ generated along it due to the orbital motion of the tether, 2) the wire provides a low-resistance path connecting different regions of the ionosphere, and 3) access to external electron and ion currents is confined to specific locations, such as the endpoint when the conductor is insulated, or collected along a length of bare tether. ${ }^{3}$ We briefly describe the principles of EDT systems using the Tethered Satellite System (TSS) as an example.

The first principle listed above, emf generation across the tether, results from the Lorentz force on the electrons in the tether as the system travels through the geomagnetic field. To determine the magnitude of this emf, we start with the Lorentz force equation for charged particles:

$$
F=q\left(\mathbf{E}+\mathbf{v}_{s} \times \mathbf{B}\right)
$$

where $q$ is the charge of an electron, $\mathbf{E}$ represents any ambient electric field (small), and $\mathbf{v}_{s} \times \mathbf{B}$ represents the motional electric field as the Shuttle traveled at a velocity $\mathbf{v}_{s}$ through the Earth's magnetic field, represented by $\mathbf{B}$. Eq. (1) can be rewritten as

$$
\mathbf{F}=q \mathbf{E}_{\text {tot }},
$$

where

$$
\mathbf{E}_{\mathrm{tot}}=\mathbf{E}+\mathbf{v}_{s} \times \mathbf{B}
$$


and represents the total electric field along the tether. In order to get the total emf generated across the tether, we must integrate $\mathbf{E}_{\text {tot }}$ along the entire length of the tether, $l$. That is, the total tether potential is

$$
\varphi_{\text {tether }}=-\int_{0}^{l} \mathbf{E}_{\text {tot }} \cdot d \mathbf{l} \approx-\int_{0}^{l} \mathbf{v}_{s}(l) \times \mathbf{B}(l) \cdot d \mathbf{l},
$$

which is negative since electrons in the tether are acted upon by the Lorentz force. Because the ionospheric plasma surrounding the EDT system is generally a good conductor, the ambient electrostatic field $\mathbf{E}$ is small and is usually ignored, i.e., $\mathbf{E} \approx 0$. The tether potential is path independent assuming a conservative resultant electric field and steady-state conditions. Thus, $\varphi_{\text {tether }}$ can be calculated knowing only the relative locations of the endpoints (separation distance and orientation) and does not depend on the position of the tether between the endpoints.

The second and third principles are related to current flow through the tether, which occurs when a connection is made between the tether's endpoints and the surrounding ionospheric plasma. This can be accomplished via passive or active means. In the passive case, the voltages and currents in the overall system distribute themselves in a self-consistent manner, which can require the endpoints to charge to high levels in order to attract enough current. Active means generally employ an electron generator of some type, such as an electron gun or hollow cathode plasma contactor. Future tether systems may employ field emitter array cathodes (FEACs), ${ }^{4}$ but much work remains before FEACs are practicable for EDT systems. With either method, current flows through the tether as shown in Figure 2. In this case, current flows up the tether because the resultant force on the electrons is downwards. After electrons are collected at the satellite, they are conducted through the tether to the Orbiter where they are ejected. Current closure occurs in the ionosphere, thus making the overall circuit complete.

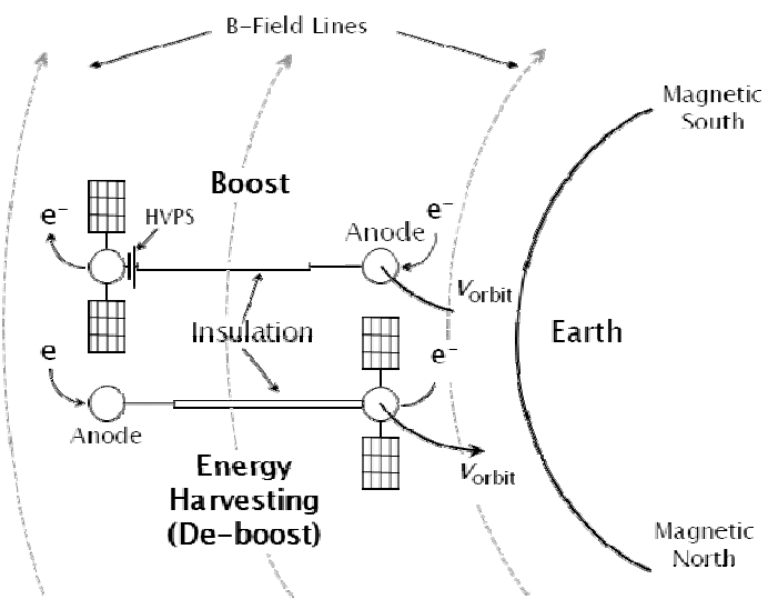

Figure 2. EDTs can be used in an "energy harvesting" mode or a "boost" mode.

Let us consider the TSS system as an example of an upwardly deployed EDT system in low Earth orbit. That is, the tether is vertically oriented, the Shuttle's orbital velocity, $v_{\text {orbit }}$, is $7.7 \mathrm{~km} / \mathrm{s}$ in an eastward direction with respect to a stationary Earth $\left(v_{\text {rot }} \approx 0.4 \mathrm{~km} / \mathrm{s}\right)$, and the geomagnetic field is oriented south to north. Since the ionospheric plasma and geomagnetic field co-rotate with the Earth, the orbital velocity should actually be in the reference frame of the Earth's rotation which yields $v_{\mathrm{s}} \approx 7.3 \mathrm{~km} / \mathrm{s}$, where $v_{\mathrm{s}}$ is the spacecraft velocity relative to the Earth's rotation. Due to the $28.5^{\circ}$ orbital inclination, the included angle between the velocity and magnetic vectors varies in a roughly sinusoidal fashion causing the tether potential to vary. With these effects, TSS- 1 achieved a peak potential just under $-60 \mathrm{~V}$ at the $267-\mathrm{m}$ tether length. ${ }^{6}$ At the longer $19.7-\mathrm{km}$ deployment of TSS-1R, this potential was close to $-3500 \mathrm{~V}{ }^{7}$ There were also variations due to tether libration and strength of the magnetic field, which varied depending on the orbital position of TSS.

If current flows in the tether element, a force is generated as given by

$$
\mathbf{F}=\int_{0}^{l} \mathbf{I}(l) \times \mathbf{B}(l) \cdot d \mathbf{l} .
$$

In self-powered mode (energy-harvesting or de-orbit mode), this emf can be used by the tether system to drive the current through the tether and other electrical loads (e.g., resistors, flywheels, batteries), emit electrons at the emitting end, or collect electrons at the opposite. In boost mode, on-board power supplies must overcome this motional emf to drive current in the opposite direction, thus creating a force in the opposite direction (see Figure 2), thus boosting the system. Thrust levels are highly dependent on applied power and typically less than $1 \mathrm{~N}$.

\section{B. Modeling Software Tools}

We have two tools available to aid in our evaluation of the energy-harvesting EDT system configurations: TEMPEST and TetherSim. 


\section{TEthered Mission Planning and Evaluation Software Tool (TEMPEST)}

In 1994, development of a tool was initiated at the University of Michigan's Space Physics Research Laboratory (UM/SPRL) in support of the reflight of the Tethered Satellite System (TSS-1R) that would allow for the simulation of the behavior of a tether system in space. The initial purpose of the TEthered Mission Planning and Evaluation Software Tool (TEMPEST) tool was to realistically predict the induced tether voltages and collected tether currents of the TSS-1R mission based on the tether system configuration, spacecraft orbital parameters, local magnetic field, and ambient plasma to aid in the planning of the mission and training of instrument operators. Very quickly TEMPEST was found to be useful not only for the TSS-1R mission for other electrodynamic tether concepts, and its feature set and capabilities have been increasing ever since. TSS -1 and TSS- $1 \mathrm{R}$ flight data were used to validate TEMPEST simulation results.

Over the years TEMPEST has been used to study various tethered systems and support tethered satellite system development and has evolved to include the following features:

- Precision Runge-Kutta 4(5) orbital propagator (with perturbations),

- Solar and Magnetic indices prediction (e.g., F10.7, $k_{p}, a_{p}$ ),

- International Geomagnetic Reference Field (IGRF) Earth magnetic field model,

- MSIS/MSISE neutral atmosphere model (valid up to 1000-km altitude),

- International Reference Ionosphere (IRI) model of ionospheric densities and temperatures (valid in the altitude range $50 \mathrm{~km}$ to $2000 \mathrm{~km}$ ),

- Simple tether libration forced harmonic mode,

- Induced electromotive force (emf) tether voltage model

- Tether current collection model for bare and insulated tethers with various plasma contactors (hollow cathodes, field emissive arrays, thermionic emitters, various collecting surfaces).

To date, TEMPEST has been used primarily for electrodynamic tether propulsion studies where orbital deboost, boost, ${ }^{8}$ as well as other orbital parameter changes were desired. ${ }^{9}$ TEMPEST can be readily utilized to simulate and evaluate the performance of tethers system for power generation with small incremental software development effort. This effort will be focused on allowing more detailed tether system configuration for power generation, as well as producing output of already available internal simulation values of interest. TEMPEST is still in development and use today at UM/SPRL, Tethers Unlimited Inc. (TUI), and ElectroDynamic Applications (EDA).

\section{TetherSim ${ }^{\mathrm{TM}}$}

TetherSim is a simulation toolset developed by Tethers Unlimited, Inc. over the past 12 years to facilitate design and analysis of space and terrestrial systems that involve tethers. TetherSim's key distinguishing feature with respect to TEMPEST is that it incorporates algorithms for simulating the dynamic behavior of a tether and the bodies connected to it. Consequently, where TEMPEST is more useful for rapid exploration of a wide engineering trade space, TetherSim is more suitable for detailed analysis of system behavior, operations, and effects on the host vehicle. The TetherSim code integrates models for orbital mechanics, tether cable dynamics, spacecraft attitude dynamics, and aircraft aerodynamics with NASA-standard environment models such as the IRI ionosphere model, MSIS neutral density model, and the IGRF magnetic field model to enable simulation of a wide range of tether systems. The models incorporated into TetherSim are summarized in Figure 3. TUI has utilized TetherSim to simulate electrodynamic drag/power generation tethers for spacecraft deorbit applications, electrodynamic propulsion tethers for orbital maneuvering ${ }^{10}$ momentum-exchange tethers for orbit transfer, ${ }^{11}$ formation-flight using tethers, and towing of tethered sensors from aircraft. It has been validated against flight data from the Plasma Motor Generator (PMG) experiment.

\section{Initial Results}

We report here our preliminary results for energy harvesting with EDTs on large-to-small (high power), nano-, and pico-satellite scales.

\section{A. Simulation of High Power EDT Power Generation}

While analytical models can provide first-order estimates of the power generation or propulsion capabilities of an EDT system, the system's performance depends upon a large number of complex, interrelated phenomena, including the dynamic behavior of the tether system and the highly nonlinear characteristics of the electrical connection between the tether and the varying ionospheric plasma. Consequently, in order to study the potential performance of an ED tether power generation system and its dependence upon system characteristics and orbital conditions, we utilized the TetherSim code (describes above) to simulate an ED tether in power generation mode. 


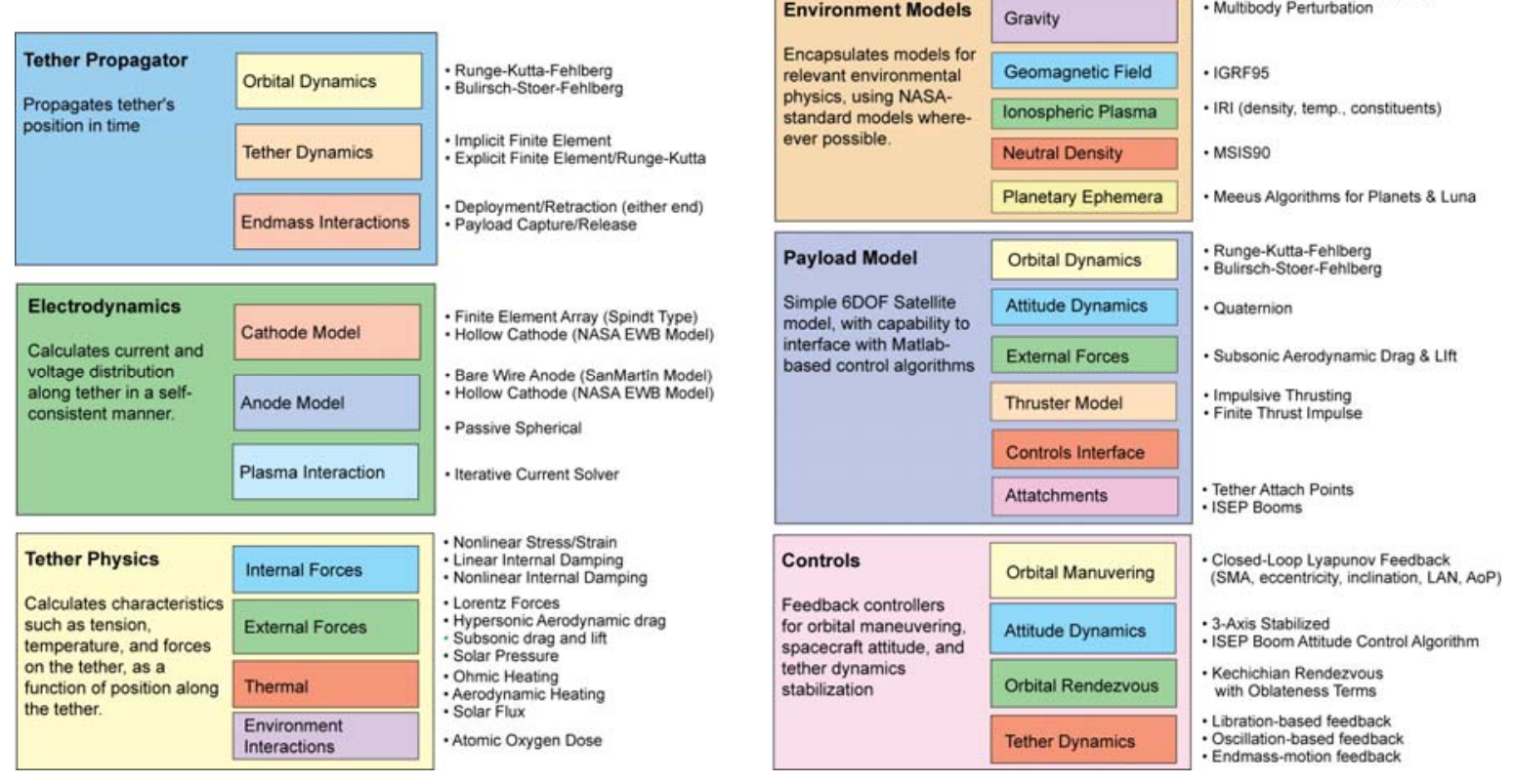

Figure 3. Overview of models incorporated into the TetherSim code.

The simulations were run using a tether with a length of $10 \mathrm{~km}$ and a conductor mass of $7.6 \mathrm{~kg}$ of aluminum. The system configuration used an insulated tether, with hollow cathode plasma contactors (HCPCs) at each end of the system to enable electrical connection to the ionospheric plasma. The tether was set into an initial orbit of $500 \mathrm{~km}, 28.5^{\circ}$ inclination, and the emf induced in the tether was allowed to drive current along the tether and through a load impedance placed in series with the tether. Figure 4 shows plots of the voltage and power dissipated across the load, tether, anode HCPC, and cathode HCPC, as well as the overall efficiency of conversion of orbital energy to electrical power in the load. The plots show that the tether system can provide conversion efficiencies on the order of $80 \%$. The power generated by the tether varies significantly over
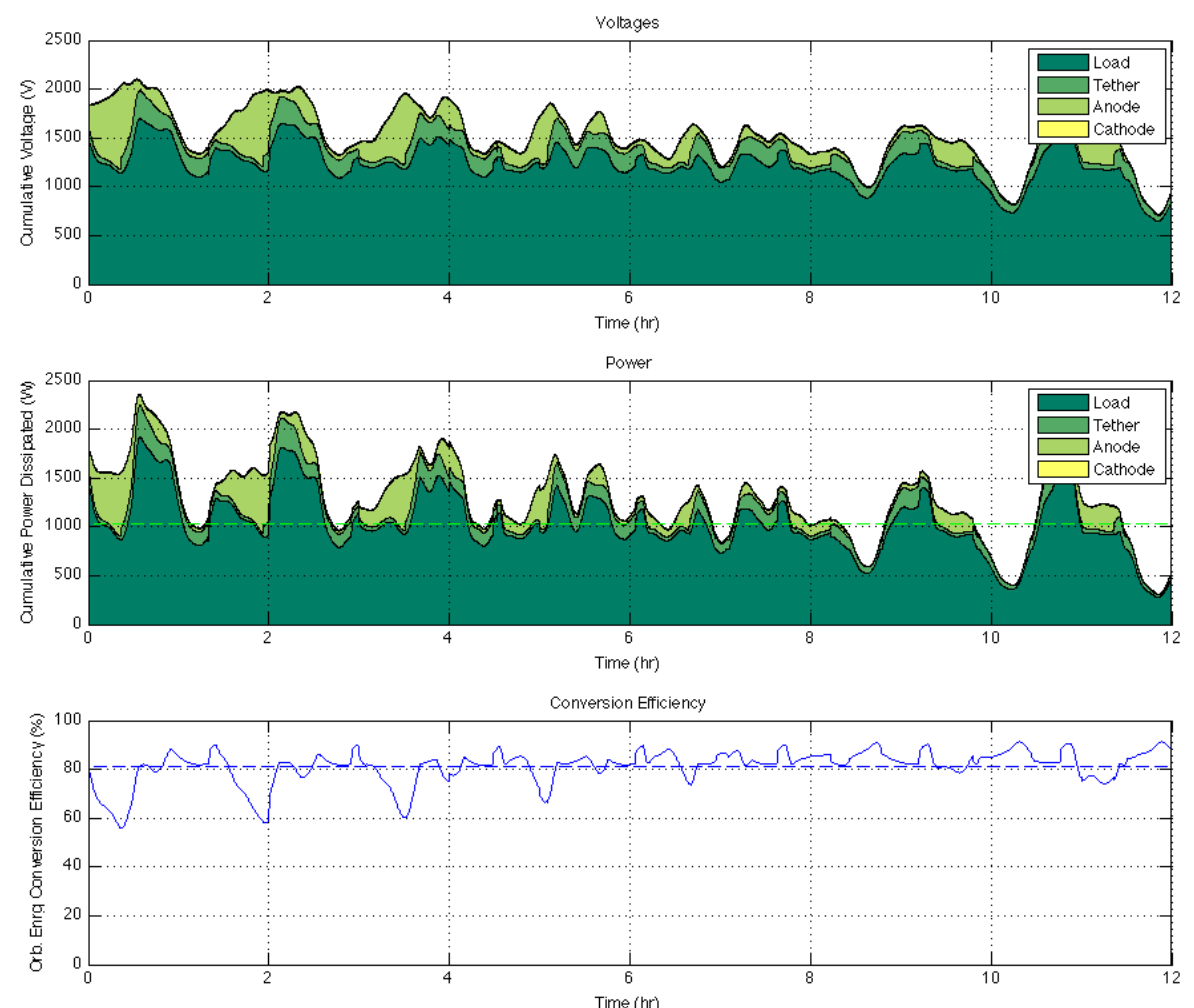

Figure 4. Plots of system voltages, power, and conversion efficiency for a 10km-long EDT system in a $500 \mathrm{~km}, 28.5^{\circ}$ orbit, simulated over a half-day period using the TetherSim code. The tether in this simulation contained $7.6 \mathrm{~kg}$ of aluminum conductor, and the system used hollow cathodes at both ends of the tether to contact the ionospheric plasma. 
the course of each orbit. This variation is due primarily to the large variations in ionospheric plasma density around the orbit. On the eclipse side of the orbit, the low plasma densities result in large voltage drops developing between the anodic HCPC and the ambient plasma in order to collect the electron current from the ionosphere. Additionally, the power generated varies over the course of multiple orbits as the Earth's magnetic field rotates with respect to the orbit plane. However, unlike a solar cell based power system, the tether is able to provide power throughout the entire orbit. The 10-km-long tether can provide an average of $1 \mathrm{~kW}$ to the load. Figure 5 shows the variation of peak, orbit average, and minimum power generation capability as the tether length is increased.

Figure 6 and Figure 7 show the dependence of the power delivered to the load and the conversion efficiency as the load impedance is varied. The variation of power with load impedance does not follow the behavior of a typical linear system. Rather, because the anode impedance varies with ionospheric density and current level in such a way that the current essentially "saturates" at a level determined by the local plasma density and the anode impedance increases very rapidly if the current is increased beyond that level, the optimum load impedance for maximum power generation is higher than would be expected if the system impedances were constant.

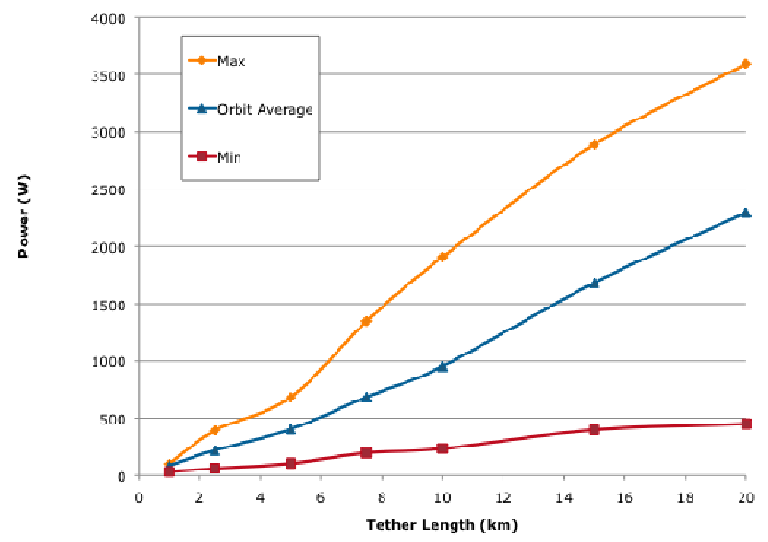

Figure 5. Variation of power generated by an electrodynamic tether upon the tether length.

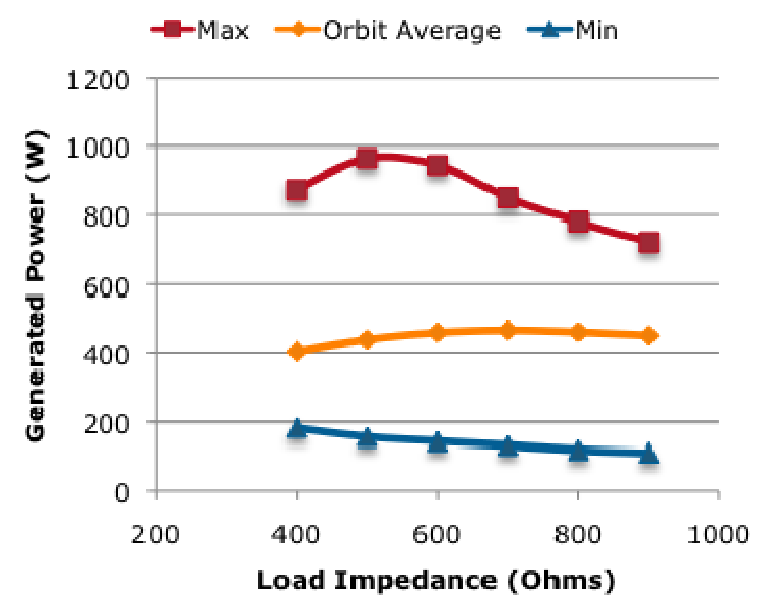

Figure 6. Variation of power generated with load impedance.

\section{B. CubeSat Energy Harvesting Concept}

In this section, we discuss some preliminary results on harvesting for satellitesreferred to as "nano" satellites, on the order of 1 to $10 \mathrm{~kg}$, though the exact size and mass cutoffs for such designations are ambiguous. The platform was chosen to be compatible with the CubeSat standard developed by California Polytechnic State University. ${ }^{12}$ The total mass of the satellite must be no more than $4 \mathrm{~kg}$ (for a 3-unit configuration). Our design allows $1.33 \mathrm{~kg}$ (essentially 1 unit) to be allocated to electrodynamic energy harvesting. This allocation includes the tether, endmass, electron emitter, and other associated hardware. All of these components will be discussed in detail.

\section{Tether}

System designers have a number of options when selecting the EDT itself. For certain applications such as energy harvesting, a bare tether will outperform one that is fully or partially insulated when in a high electron density plasma. ${ }^{13}$ The opposite is true for lower electron densities where a partially insulated tether is preferred. No insulating materials were considered for this study because of their added mass.

Two conducting materials have been considered so far: copper and aluminum. A low resistivity tether, such as copper, is preferred for optimum current flow, but is not always the best choice. For a CubeSat to harvest as much 
energy as high efficiency solar panels mounted on all six faces, a minimum of $1300 \mathrm{~m}$ of 25 AWG is needed for reasons that are discussed below. This amount of aluminum tether has a mass of $0.558 \mathrm{~kg}$ where an equally long and thin copper tether would have a mass of $1.848 \mathrm{~kg}$, already exceeding the $1.33 \mathrm{~kg}$ mass allocation limit. Therefore, aluminum was chosen for this study because of its relatively high conductivity and low mass. The tether composition is determined by the power needed for the mission and by mass limitations. The trade-offs are discussed in the Section III.B.8 below.

\section{Endmass}

The endmass is a passive or active mass attached to the opposite end of the EDT from the satellite. If the endmass is passive, its functions are to help in the deployment of the tether and provide tension after full deployment. An active endmass will do them same as the passive but can be fitted with a plasma contactor to aid in the current closure loop through the ionosphere. CubeSat size and weight restrictions require the use of a passive endmass. TUI has developed the "nano-Terminator," which has a mass less than $0.065 \mathrm{~kg}$ and is about the size of a D-cell battery. ${ }^{14,15}$

\section{Electron Emitter}

An electron emitter, or "plasma contactor", assists the flow of electrons between the conducting tether and the ionospheric plasma. There are three types of active emitters: hollow cathode plasma contactors (HCPCs), thermionic cathodes (TCs), and field emitter arrays cathodes (FEACs). ${ }^{5}$ HCPCs are too large to fit into a CubeSat in part because there is no room to store the required ionizing gas used in its operation. FEACs would be a better choice, though more development is needed before their use can be realized. Thermionic cathodes can be packaged smaller and are simpler to construct and operate making it the preferred choice for the CubeSat standard. The thermionic cathode emits electrons when heated like a filament in a light bulb. The flow of electrons into the surrounding plasma at the emitter promotes flow of electrons into the system along the bare tether. Thus, energy is brought "harvested" into the system.

The power harvesting simulations performed via TEMPEST incorporated a HCPC model, even though our platform would use a TC. It is believed that the two contactors would perform similarly for this system, and further research will include a TC contactor model in TEMPEST.

\section{Associated Hardware}

An EDT system will also need a DC/DC converter and current regulating hardware to convert the harvested energy into a usable form for charging a battery or operating a scientific payload.

\section{Assumptions}

There are some assumptions made about the EDT system and the solar panel system. Regarding the EDT system, it is assumed that there are no librations of the tether. It is modeled to behave like a rigid tether that is always aligned with the local gravity gradient. The solar panel system is assumed to be in one of two positions; maximum projected surface area to the Sun and the minimum. The maximum surface area of a CubeSat that can be exposed to the sun is $0.0436 \mathrm{~m}^{2}$. The minimum surface area occurs when the smallest face, $0.01 \mathrm{~m}^{2}$, is aligned with the Sun. A typical CubeSat will rotate as it orbits the Earth. Therefore, the instantaneous surface area will be less than or equal to these extremes. The exact rotation of the CubeSat is not considered in this study, only the extremes.

\section{Mission Lifetime}

A satellite equipped with an EDT harvesting system will lose altitude while in harvesting mode. This is due to a Lorentz force interaction between the current flowing in the tether and the geomagnetic field as described in Section II above. The larger the current the faster the satellite system will de-orbit. A CubeSat configured according to our platform definition will de-orbit after 16 days of continuous harvesting (Figure 8). The mission lifetime can be extended by harvesting energy intermittently. As the satellite looses altitude and gets closer to the surface of the Earth it is exposed to an increased electron density. This increase in electron density will yield higher power (Figure 9). Below $300 \mathrm{~km}$ the electron density decreases. This occurs on day 15.

Over the course of just one solar day, a satellite at an altitude of $500 \mathrm{~km}$ will orbit approximately 15 times. During an orbit the electron density changes due to solar effects, thus affecting the amount of power that can be harvested (Figure 9). The maximum power available for day 16, using $1300 \mathrm{~m}$ of bare aluminum, is seen to be $44 \mathrm{~W}$. This maximum will be lower at the beginning of the mission because of the before mentioned electron density change. 


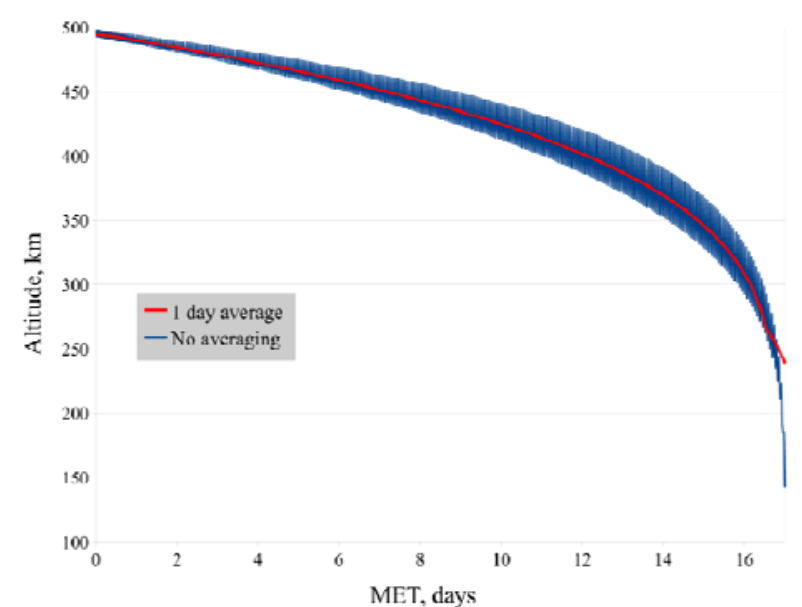

Figure 8. Altitude as a function of mission elapsed time. 1300 meters, $0.558 \mathrm{~kg}$, aluminum tether.

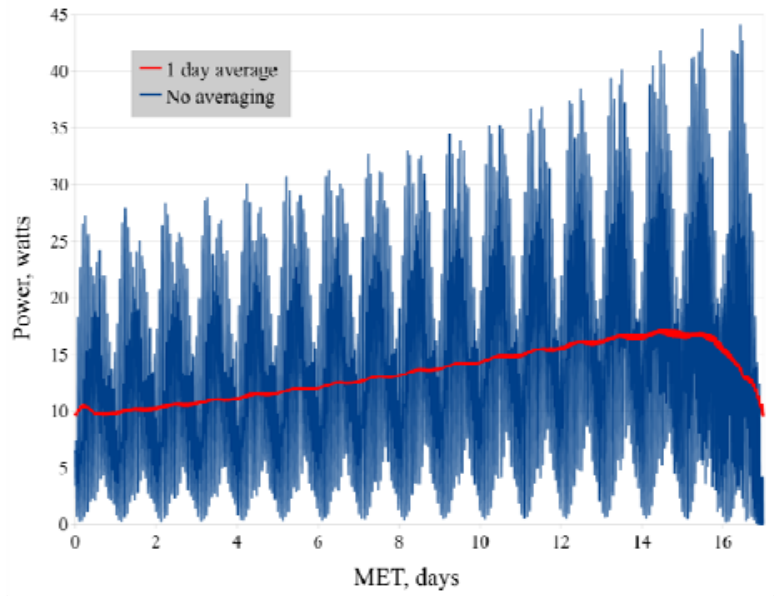

Figure 9. Power delivered to the load resistor as a function of mission elapsed time. 1300 meters, 0.558 kg, aluminum tether.

\section{Average Power}

Because the power is not constant during an orbit, it is necessary to consider the time frame for which the power will be needed. A moving average program with a user defined window was developed for TEMPEST. This program acts like a smoothing function and makes it possible to ascertain the maximum average power available over a given period of time. For example, for a 10-minute timeframe, the approximate time that a satellite could communicate directly to a ground station, the maximum average power was $42.0377 \mathrm{~W}$ (Figure 10). The maximum nonaveraged power was $44.0828 \mathrm{~W}$. This implies that the satellite could have access to about $44 \mathrm{~W}$ for about one minute, or about $42 \mathrm{~W}$ for 10 minutes. For day one of

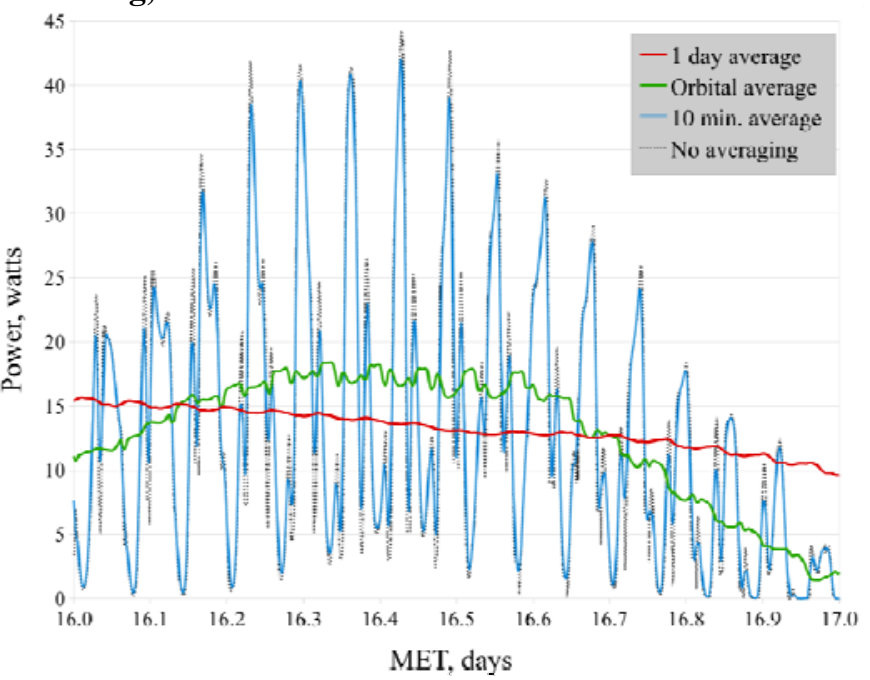

Figure 10. Power and various averages as a function of MET.

the mission, the maximum non-averaged power was $27.1738 \mathrm{~W}$. If a satellite needs to harvest energy for an entire orbit, it will only have around $14 \mathrm{~W}$ available on average.

\section{Total Energy}

The previous discussion considers using only the EDT or solar panel as an energy source and omits the possibility of using a battery as a means of producing high power output. Both EDT and solar panel systems can use battery-stored energy in bursts to produce as much power as the battery will output and the system can thermally withstand. However, the EDT has the possibility of harvesting more energy (Figure 11), allowing for higher duty cycles. In the figure, the total harvestable energy using various tether lengths and, therefore, mass, is compared to the best and worst case scenario using solar panels alone. A tether length of $1300 \mathrm{~m}$, which corresponds to $0.558 \mathrm{~kg}$ of aluminum wire, would harvest the same amount of energy as the best case scenario for solar panels. To guarantee an increase in total energy harvested, one needs to use a tether longer than this amount; however, system size and mass restrictions limit the length. If $0.687 \mathrm{~kg}$ and $1600 \mathrm{~m}$ of tether can be allocated to the system, then the EDT could harvest over $50 \%$ more energy per day. This energy figure will be smaller towards the beginning of the mission and be larger towards the end due to the altitude decrease and the associated electron density changes.

The plateau at $64 \%$ of the day in Figure 11 represents the percent of time the system spends in the Earth's shadow. Solar panels cease harvesting energy while the EDT continues. While in the shade, the effectiveness of the EDT changes due to changes in the electron density, yet it still continues to harvest energy. Figure 12 overlays the 
power available to the EDT system with the time spent in the shade. The available power decreases when the satellite is the Earth's shadow, but does not go to zero as in the case of solar panels.
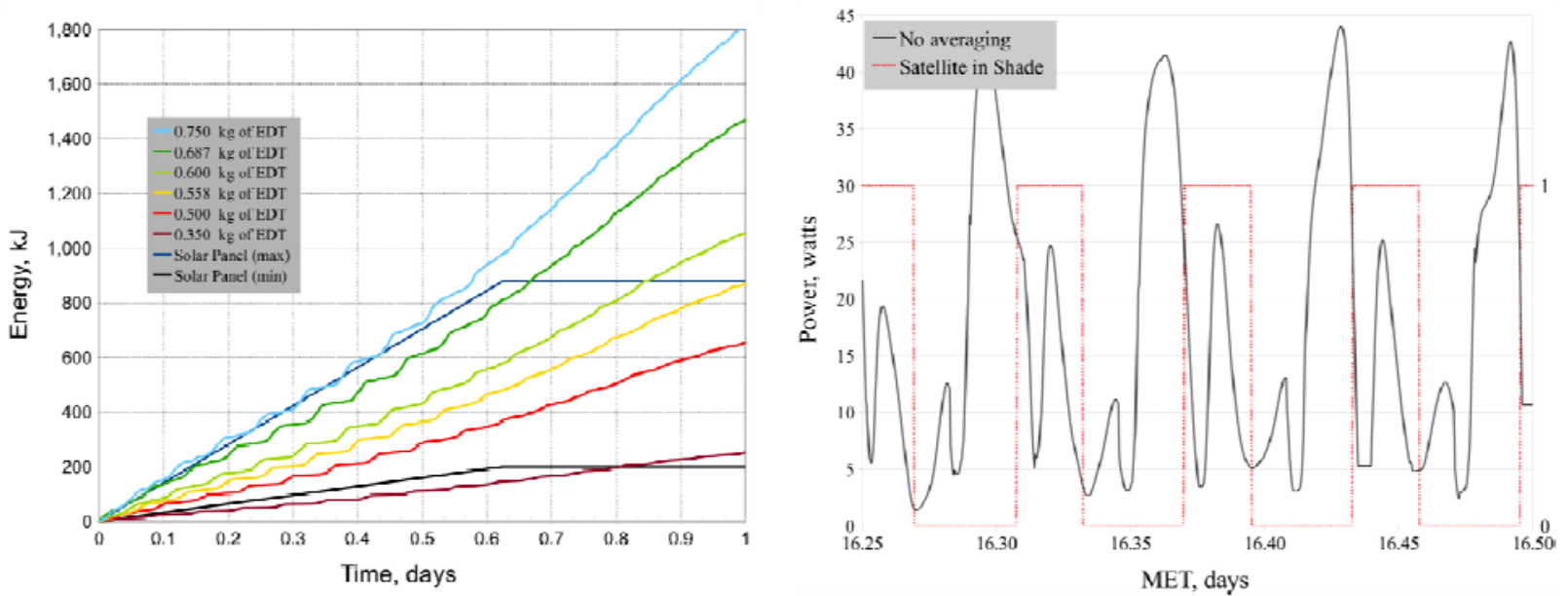

Figure 11. Total energy vs. time.

The total energy capable of being harvested depends on the system configuration. Longer tethers yield more power, but weigh more. Figure 13 shows the relationship between tether mass and the total energy that can be harvested during one day. Data for both 24 and 25 AWG are shown to illustrate the benefit of using a thinner tether to harvest more energy for the same amount of mass. There is a crossover point at $0.558 \mathrm{~kg}$ where total energy from the tether is equal to the best-case scenario for solar panels. System designers can look at the mass allocation for the EDT and the power budget to choose a suitable length. This is the mass-to-total-energy trade-off.

If a hybrid system of EDT and solar panels is needed because the available power from solar panels is not sufficient for the application, then the mass (and therefore length) of tether could be chosen based on the energy deficit. For example, a

Figure 12. EDT Power and when the satellite is in the Earth's shadow vs mission elapsed time.

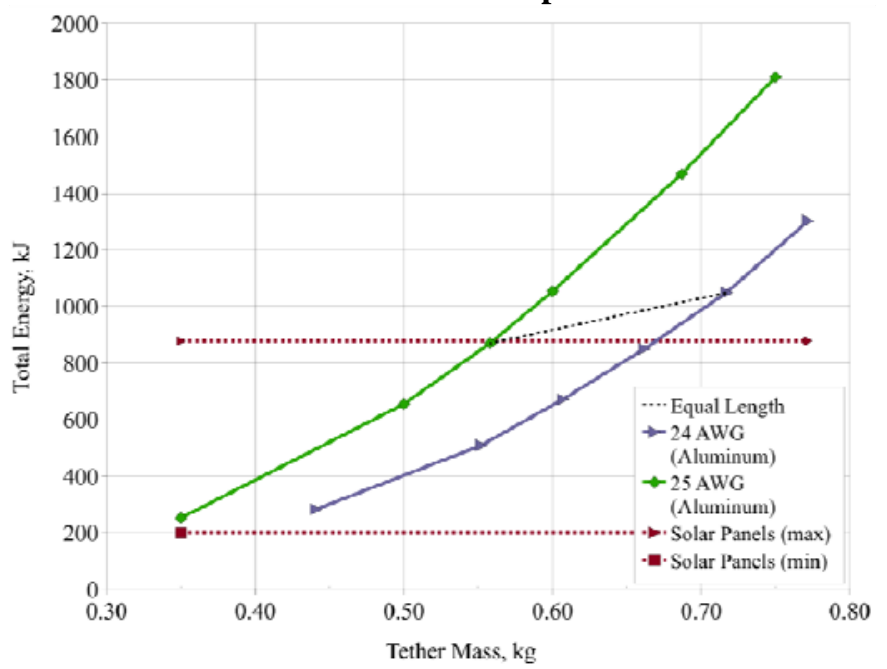

Figure 13. Trade-off between total energy harvestable and tether mass.

mission that requires $1,200 \mathrm{~J} /$ day would require either more solar panel surface area or an addition of approximately $0.50 \mathrm{~kg}$ of an EDT system.

\section{EDTs for ChipSats and Nanospacecraft}

The recent interest in nanospacecraft $(1-10 \mathrm{~kg})$ over the past decade has spawned an interest to assess even smaller spacecraft. Based on integrated circuit technology, the potential of highly miniaturized spacecraft at the levels of hybrid integrated circuits and fully monolithic computer chips is being investigated. These small "chip" satellites, or ChipSats, would have the potential of leveraging the technology development of highly integrated, highly capable microelectromechanical systems (MEMS) and could be substantially less expensive to bring into orbit. ChipSats are thus a new generation of lightweight (on the order of milligrams to grams), small spacecraft that can be released in swarms, for example, to provide a unique form of data collection. ${ }^{16}$

Early ChipSat concepts have no propellant and a low ballistic coefficient, so ChipSats maintain their orbit for no more than a few days in low Earth orbit. Table 1 shows the rough estimates of the expected lifetimes of a 3-unit CubeSat, an 8-g ChipSat, and a 7.5-mg ChipSat. The assumption is made that the satellites are orbiting during a solar maximum, the drag coefficient for the satellites is 2.2 , and the side with the largest cross sectional area is oriented parallel to the satellites' velocities. This orientation reduces the effect of atmospheric drag. 
Table 1. Rough estimate of satellite's lifetime due to atmospheric drag

\begin{tabular}{|c|c|c|c|}
\hline Altitude & 3-kg CubeSat & 8-g ChipSat & 7.5-mg ChipSat \\
\hline $300 \mathrm{~km}$ & $\sim 1$ month & $\sim 1$ month & several days \\
\hline $400 \mathrm{~km}$ & several months & several months & several weeks \\
\hline $500 \mathrm{~km}$ & $\sim 1$ year & $\sim 1-2$ years & several months \\
\hline
\end{tabular}

Flexible or semi-rigid EDTs can be used to meet the challenge of maintaining ChipSat orbit and harvesting energy without contributing significantly to the ChipSat mass, required onboard power, or atmospheric drag relative to the thrust. Most previous EDT work accounts for tethers that are kilometers long. However, several meter-long EDTs are considered for ChipSat systems in order to keep the ChipSat's mass and size small.

There is a trade-off between EDT current and length required for the drag make-up force. Figure 14 shows the required current for a range of EDT lengths at an altitude of $500 \mathrm{~km}$. Each current-length product (where the magnetic field is estimated to be 0.3 gauss) represents a Lorentz force that exactly balances the satellite's drag force. The assumptions made for estimating the satellites' lifetimes are also used in the drag force calculation, except here the side with the largest cross sectional area is oriented perpendicular to the satellite's velocity. This orientation is used to show the range of EDT lengths and currents required when the satellite is in an orientation that increases atmospheric drag force.

The current required to maintain the orbit of the $7.5-\mathrm{mg}$ ChipSat at $500 \mathrm{~km}$ is only $\sim 70 \mu \mathrm{A}$ for a $5-\mathrm{m}$ EDT. The microampere currents considered are

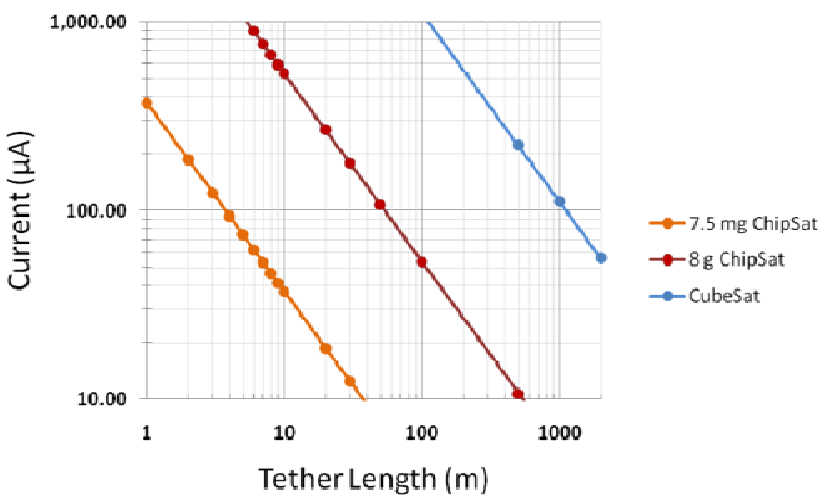

Figure 14. Minimum tether current-length product for $500-\mathrm{km}$ orbital maintenance.

suitably small and the EDT length is on the order of meters, so this shows that the EDT may be a solution for maintaining the orbit of small satellites. However, the Lorentz force calculation considered here does not take into account the additional drag introduced by the EDT or a current collector on the opposite side of the tether. Further analysis incorporating these factors is being done to provide another estimate of EDT currents and lengths for ChipSat.

\section{Conclusion}

An electrodynamic tether can be used by satellites to harvest energy from orbital potential by using the Lorentz force interactions with the geomagnetic field. The amount of energy that can be harvested depends on the length of the tether, the type of tether, and the configuration of the plasma contactors. Satellite size sets the bounds for the configuration of the EDT system and, therefore, the magnitude of the energy budget.

Large satellites have the potential to harvest as much as kilowatts at the load. Small EDT systems the size of CubeSats have the potential to harvest $50 \%$ more energy than solar panel systems alone and can produce over 40 watts-average power useful during, for example, a 10-minute ground station overpass. EDT systems benefit very small, ChipSat-sized satellites by enabling them to maintain orbit without a significant contribution to the satellites' size and mass or the need for expendable propellant.

EDTs provide a unique solution to satellites' energy and propulsion needs throughout the size and mass spectrum of current and future technology. This research demonstrates the benefits, and necessary trade-offs, system designers will encounter when considering the energy budget, which is critical to satellite performance, and demonstrates the value of further EDT investigation.

\section{Acknowledgment}

We gratefully acknowledge support from AFOSR grant FA9550-09-1-0646.

\section{References}

${ }^{1}$ Gilchrist, B.E., Thompson, D.C., Raitt, W.J., Voronka, N., Agüero, V., Williams, S.D., Bilén, S.G., and Bonifazi, C., "Measurements of Current and Voltage in the TSS-1R System Using Passive Current Closure and Electron Beam Emission from the Orbiter Structure,” 1996 Spring AGU Meeting, Baltimore, MD, May 1996. 
${ }^{2}$ Banks, P.M., "Review of Electrodynamic Tethers for Space Plasma Science," J. Spacecraft and Rockets, Vol. 26, No. 4, pp. 234-239, 1989.

${ }^{3}$ Sanmartín, J.R., Martinez-Sánchez, M., and Ahedo, E., "Bare Wire Anodes for Electrodynamic Tethers," J. Prop. and Power, Vol. 9, No. 3, pp. 353-360, 1993.

${ }^{4}$ Morris, D.M., Gilchrist, B.E., Gallimore, A.D., and Jensen, K., "Developing Field Emitter Array Cathode Systems for Electrodynamic Tether Propulsion," AIAA Paper 2000-3867, 2000.

${ }^{5}$ Fuhrhop, K., "Theory and Experimental Evaluation of Electrodynamic Tether Systems and Related Technologies," Ph.D. Thesis, University of Michigan, 2007.

${ }^{6}$ Agüero, V., Banks, P.M., Gilchrist, B., Linscott, I., Raitt, W.J., Thompson, D., Tolat, V., White, A.B., Williams, S., and Williamson, P.R., "The Shuttle Electrodynamic Tether System (SETS) on TSS-1," Il Nuovo Cimento, Vol. 17C, No. 1, pp. 4965, 1994.

${ }^{7}$ Williams, S.D., Gilchrist, B.E., Agüero, V.M., Indiresan, R.S., Thompson, D.C., and Raitt, W.J., "TSS-1R Vertical Electric Fields: Long Baseline Measurements Using an Electrodynamic Tether as a Double Probe," Geophysical Research Letters, Vol. 25, No. 4, pp. 445-448, 1998.

${ }^{8}$ Fuhrhop, K., Gilchrist, B.E., Bilén, S.G., and Voronka, N.R., "System Analysis of the Expected Electrodynamic Tether Performance for the ProSEDS Mission," AIAA 2003-5096, 39th Joint Propulsion Conference and Exhibit, Huntsville, AL, July 20-23, 2003.

${ }^{9}$ Matthew, A, and Voronka, N., "Control of a Multi-Beam Electrodynamic Spacecraft Propulsion System," 2008 American Control Conference, June 11-13 2008, Seattle, WA.

${ }^{10}$ Hoyt, R.P., Slostad, J.T., and Voronka, N.R., "The Microsatellite Propellantless Electrodynamic Tether (PET) Propulsion System," 52nd Joint Army Navy NASA Air Force (JANNAF) Propulsion Meeting, Las Vegas, NV, 12-13 May 2004.

${ }^{11}$ Hoyt, R.P., Slostad, J.T., Frank, Scott S., and Voronka, N.R. "Momentum-Exchange/Electrodynamic Reboost (MXER) Tethers for Low-Cost Orbit Transfer," 52nd Joint Army, Navy, NASA, Air Force (JANNAF) Propulsion Meeting, Las Vegas, NV, 12-13 May 2004.

${ }^{12}$ CubeSat Design Specification Rev. 12, The CubeSat Program, Cal Poly SLO.

${ }^{13}$ Fuhrhop, K R., and Gilchrist, B E., "Electrodynamic Tether System Analysis Comparing Various Mission Scenarios," $41^{\text {st }}$ AIAA/ASME/SAE/ASEE Joint Propulsion Conference \& Exhibit, 10-13 July, 2005

${ }^{14}$ Hoyt, R., "Space Debris Mitigation Technologies," Tethers Unlimited, Inc.,

${ }^{15}$ Voronka, N., and Hoyt, R., "Bare Tether Electrodynamic Propulsion without the Use of Endbody Plasma Contactors," Joint Propulsion Conference, 2005, AIAA 2005-4290.

${ }^{16}$ Atchison, J.A. and Peck, M., “A Millimeter-Scale Lorentz-Propelled Spacecraft," AIAA 2007-6847, AIAA Guidance, Navigation and Control Conference and Exhibit, 20-23 August 2007. 